\title{
Cigarette smoking and bone mineral density in the elderly
}

\author{
P Egger, S Duggleby, R Hobbs, C Fall, C Cooper
}

\begin{abstract}
Objective - To test the hypothesis that cigarette smoking is associated with reduced bone mineral density in elderly men and women.

Design - Cross sectional study.

Subjects - These comprised 224 men and 186 women aged 61-73 years, born and resident in East Hertfordshire.

Measurements - Lumbar spine and femoral neck bone mineral density (BMD) were determined by dual energy $x$ ray absorptiometry.

Results - After adjusting for potential confounding variables, men who were current smokers were found to have a lumbar spine BMD that was $7 \cdot 3 \%(95 \%$ CI $0.4,14.2)$ lower than men who had never smoked. Similarly, women who were current smokers had a BMD value that was $\mathbf{7 \cdot 7 \%}$ $(95 \% \mathrm{CI} 0.3,15.6)$ lower than in women who had never smoked. The difference at the femoral neck was smaller and not statistically significant. For both men and women, each decade of smoking was associated with a reduction of $0.015 \mathrm{~g} / \mathrm{cm}^{2}$ in BMD at the lumbar spine $(95 \%$ CI: men $0.002,0.027$; women $0.003,0.028$ ). At the femoral neck the reduction in BMD was $0.011 \mathrm{~g} / \mathrm{cm}^{2}(95 \% \mathrm{CI} 0.003,0.020)$ for men and $0.004 \mathrm{~g} / \mathrm{cm}^{2}(95 \% \mathrm{CI}-0.003,0.012)$ for women with each decade of smoking.

Conclusions - The results show an adverse effect of smoking on BMD which was most noticeable at the lumbar spine, and seemed stronger in men than women. This effect could not be explained by differences in life style between smokers and nonsmokers.
\end{abstract}

The MRC

Environmental

Epidemiology Unit, Southampton General

Hospital,

Southampton

SO16 6YD

P Egger

$S$ Duggleby

C Fall

C Cooper

Department of Radiology, Queen

Elizabeth II Hospital

Welwyn Garden City, Herts AL7 4HQ

R Hobbs

Correspondence to: Dr C Cooper.

Accepted for publication September 1995
( 7 Epidemiol Community Health 1996;50:47-50)

Hip fracture is a major public health problem in the elderly and strategies to reduce the incidence are urgently required. ${ }^{1}$ Epidemiological studies suggesting an association between cigarette smoking and hip fracture have led to the conclusion that measures to reduce cigarette smoking can be expected to reduce hip fracture rates in the general population. ${ }^{2}$ The pathophysiology of hip fracture entails an interplay between several factors that influence bone mineral density (BMD) and falling, ${ }^{3}$ the extent to which smoking modifies these ultimate determinants of fracture remains uncertain. Studies of the relationship between cigarette smoking and BMD are inconsistent, ${ }^{4}$ and have tended to focus on postmenopausal women. We explored this issue in a population sample of elderly men and women.

\section{Subjects and methods}

We studied 224 men and 186 women aged 63-73 years born in East Hertfordshire between 1920 and 1930 and still resident in the county. They were enrolled in a longitudinal study examining the relationship between growth in infancy and the subsequent risk of coronary heart disease and osteoporosis. In order to select them, we traced all men who were born during 1920-30 and all women born during 1923-30 in the six districts of East Hertfordshire, and who still lived there. Because of the change of name on marriage, it is difficult to trace women born before 1923. The 370 men and 285 women who were traced as still living in East Hertfordshire took part in a study of cardiovascular risk factors, which included measurements of glucose tolerance; plasma clotting factor concentrations, and blood pressure ${ }^{56}$ For the present study we aimed to recruit 200 men and 200 women. All 285 women, and 300 men chosen randomly from the 370 men in the previous study, formed our sample and were contacted by letter. A total of $186(65 \%)$ women and $224(75 \%)$ men completed the study.

All subjects completed a structured, interviewer administered questionnaire. This included questions on socioeconomic status, medical history, cigarette smoking, alcohol consumption, dietary calcium intake, physical activity, and reproductive variables in women.

BMD was measured in each subject by dual energy $x$ ray absorptiometry at the lumbar spine and femoral neck using a Hologic QDR 1000 instrument. Results were expressed as BMD in grams of ashed calcium hydroxyapatite for each site. Measurement precision was assessed by six replicate measurements in each of 10 subjects: expressed as the coefficient of variation (SD/mean), it was $1 \cdot 1 \%$ for lumbar spine BMD and $1 \cdot 8 \%$ for femoral neck BMD.

\section{Results}

The characteristics of the study population in relation to smoking status are shown in table 1. Men and women who were current smokers tended to be less active than those who had never smoked or were ex-smokers. Women who smoked also had lower dietary calcium intakes than never or ex-smokers. 
Table 1 Characteristics of the study population by smoking status (median (interquartile range))

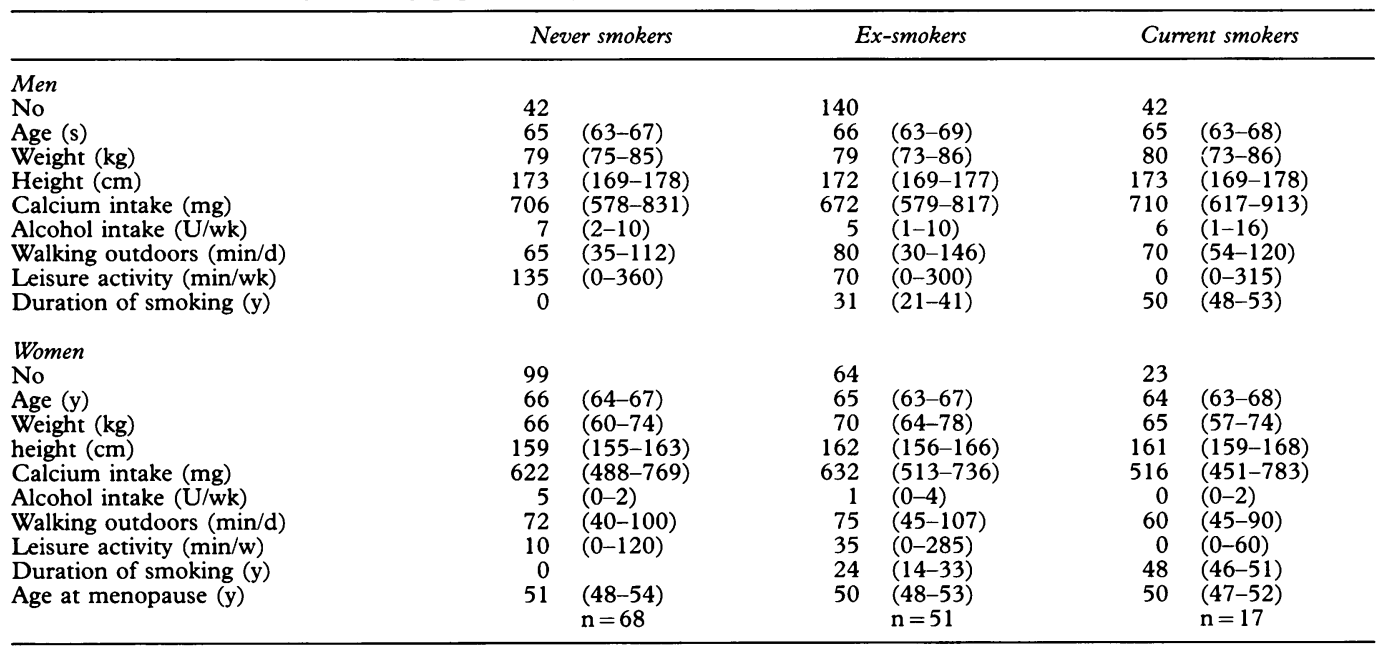

Figure 1 shows the mean lumbar spine and femoral neck BMD values among men and women who were current, previous and never smokers. There was a consistent negative association between smoking and BMD at both sites. The smoking effect was most noticeable at the lumbar spine, and seemed stronger in men than women. Using a test for linear trend, the effect was statistically significant $(p<0.05)$ at the lumbar spine among men, but not among women.

Table 2 shows the mean BMD in the three smoking categories after adjusting for age, weight, height, calcium intake, alcohol consumption, and physical activity, and additionally for age at menopause among women. It shows that the adverse effect of smoking remained after adjusting for these potential
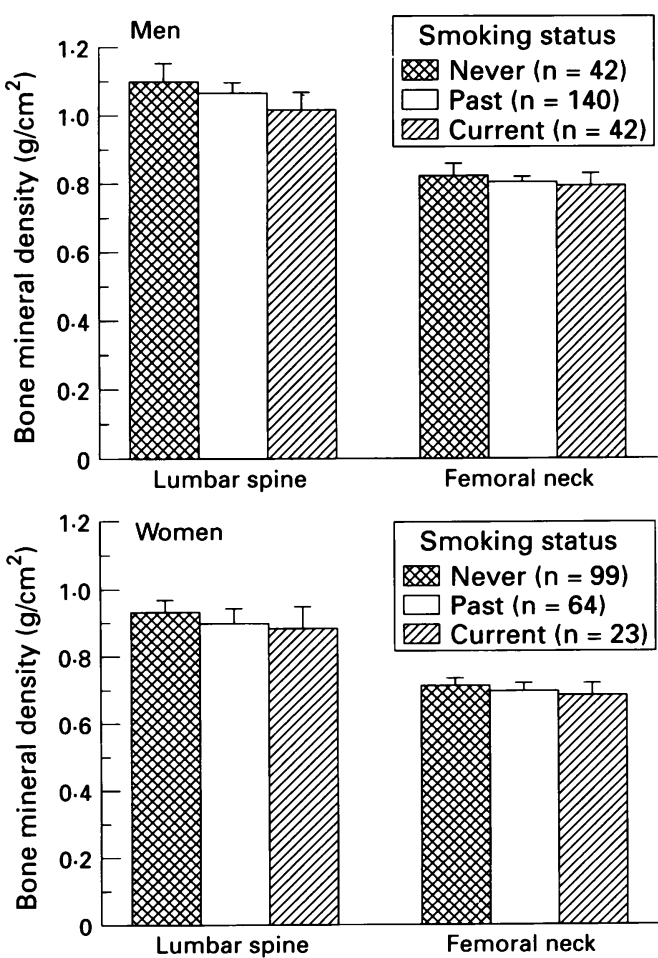

Figure 1 Cigarette smoking and bone density in men and women. Bars show mean values with the upper half of the $95 \% C I$.
Table 2 Mean bone density (BMD) in relation to smoking status*

\begin{tabular}{llll}
\hline & Never smokers & Past smokers & Current smokers \\
\hline Men & & & \\
Lumbar spine & 1.12 & 1.07 & 1.04 \\
Femoral neck & 0.87 & 0.84 & 0.84 \\
& & & \\
Women & & 0.90 & 0.89 \\
Lumbar spine & 0.97 & 0.73 & 0.73 \\
Femoral neck & 0.76 &
\end{tabular}

* Mean BMD values refer to men and women in the middle tertile of their distributions of age, weight, height, calcium intake, alcohol consumption and physical activity ( + menopausal age, for women)

confounding variables in a multiple linear regression. Men who never smoked had a greater spinal BMD $\left(0.08 \mathrm{~g} / \mathrm{cm}^{2}\right)$ and a greater hip $\operatorname{BMD}\left(0.03 \mathrm{~g} / \mathrm{cm}^{2}\right)$ than men who were current smokers. The corresponding differences in women were $0.08 \mathrm{~g} / \mathrm{cm}^{2}$ and $0.03 \mathrm{~g} / \mathrm{cm}^{2}$ respectively. Figure 2 expresses these data as the percentage reductions in BMD between current and never smokers. The difference was statistically significant $(p<0.05)$ for men at the lumbar spine but not for women. The differences at the femoral neck were not significant in either sex.

To examine the influence of duration of smoking, this variable was modelled as a continuous variable through multiple linear regression, adjusting for the same potential

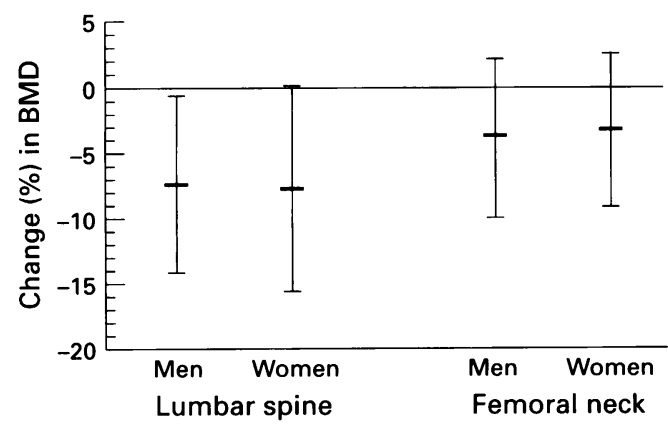

Figure 2 Mean difference in bone density (\% with 95\% CI) between current smokers and never smokers. These values are adjusted for age, weight, height, alcohol consumption, calcium intake and physical activity (plus menopausal age, for women). 
Table 3 Duration of smoking and bone density

\begin{tabular}{llc}
\hline Sex/skeletal site & \multicolumn{2}{l}{ Mean change in $B M D\left(\mathrm{~g} / \mathrm{cm}^{2}\right.$ per decade of smoking) $(95 \% \mathrm{CI})^{*}$} \\
\cline { 2 - 3 } & Whole sample & Ever smokers only \\
\hline Men & $-0.015(-0.027,-0.002)$ & $-0.012(-0.019,0.006)$ \\
Lumbar spine & $-0.011(-0.020,-0.003)$ & $-0.014(-0.026,0.002)$ \\
Femoral neck & & \\
Women & $-0.015(-0.028,-0.003)$ & $-0.003(-0.025,0.018)$ \\
Lumbar spine & $-0.004(-0.012,-0.003)$ & $0.004(-0.008,0.017)$ \\
\hline
\end{tabular}

*Values are adjusted for age, weight, height, alcohol consumption, calcium intake and physica activity ( + menopausal age for women).

confounding variables. Table 3 shows the mean difference in BMD for each decade of smoking in the entire study sample and in ever smokers when analysed alone. When the entire sample was included in the analysis, there was a negative association between duration of smoking and BMD at both sites among both men and women. The relationship was most pronounced at the spine, and was statistically significant $(p<0.05)$ at both sites among men, and at the spine among women. When the analysis was confined to ever smokers, the trends were similar, but the smaller numbers led to reduced precision in the estimates, with consequent widening of the $95 \%$ confidence intervals (95\% CI). The subjects who reported ever smoking had also been asked the maximum daily number of cigarettes which they had ever smoked. This was used to estimate the number of packyears smoked, but the variable was not associated with BMD at either site among men or women. There was no threshold apparent in the relationship of smoking duration, or age at stopping smoking, to $\mathrm{BMD}$.

\section{Discussion}

These data suggest that cigarette smoking is associated with reduced bone density among elderly men and women. Although this effect is most noticeable at the lumbar spine, it is also apparent at the proximal femur and cannot be explained on the basis of confounding by body build, alcohol consumption, physical activity, or diet in men and, additionally, by menopausal age in women.

We studied a series of men and women aged 61 to 73 years who lived in a rural county of south east England and who were not institutionalised. They were selected as part of an epidemiological study relating growth in infancy to the subsequent risk of osteoporosis, and were therefore a selected group, having been born and still living in the county. However, the prevalence of cigarette smoking in the sample was similar to that reported in randomly obtained population samples such as that used by the general household survey. ${ }^{7}$ Although biases due to response and misclassification might have influenced our results, their effects would tend to obscure any association of smoking with BMD.

We have previously shown that cigarette smoking is a risk factor for hip fracture in elderly men and women. ${ }^{89} \mathrm{~A}$ population based study comparing 300 men and women with hip fractures with 600 age and sex matched community controls showed a doubling of hip fracture risk among cigarette smokers. ${ }^{8}$ This increase in risk was confirmed in a British cohort study where 1400 elderly men and women were followed prospectively for 14 years to identify subsequent hip fractures. ${ }^{9}$ However, the reason for the relationship between smoking and hip fracture risk remains contentious. Cigarette smokers may be more prone to falling, may have reduced bone density, or both. Furthermore, smokers may differ from nonsmokers in weight, age at menopause, alcohol consumption, diet, and physical activity. ${ }^{10}$ All of these associations could potentially confound a relationship beween smoking and bone density.

Early studies of the relationship between smoking and bone density utilised single photon absorptiometry at the distal forearm..$^{41-13}$ Although several such studies have been performed, their results are inconsistent and, where smoking effects have been observed, these have not been carefully adjusted for the influence of other potential confounding variables. ${ }^{4}$ In general, these studies have suggested little influence of cigarette smoking on appendicular bone density in premenopausal and perimenopausal women, but a more pronounced adverse effect among women aged above 60 years. The few studies in men have also been inconsistent.

These data have recently been supplemented by studies using dual photon absorptiometry or dual energy $x$ ray absorptiometry to estimate bone mineral density at the lumbar spine and hip. Krall et al $^{14}$ estimated the effect of smoking on axial bone mineral density, rates of bone loss and fractional whole body retention of calcium in $\mathbf{3 2 0}$ women enrolled in a two year trial of calcium supplementation. They observed lower femoral neck and spine bone density values among smokers than non-smokers, although the differences did not attain statistical significance. Calcium retention was found to be lower in the smokers and seemed to contribute to the statistically significant increase in appendicular bone loss rate observed in this subgroup. Slemenda $e t a l^{15}$ studied the relationship between smoking and bone loss in a series of 84 perimenopausal and postmenopausal women. They found that heavy cigarette smokers had significantly lower radial and spinal BMD than light or non-smokers (who did not differ from each other). The effect of cigarette smoking on bone loss remained significant after adjusting for obesity. However, cigarette smokers did not seem to lose bone faster than non-smokers in this study and the adverse consequence of smoking seemed to be present by the time of menopause. These observations are in accordance with a recent twin study in which every 10 pack years of smoking was found to be associated with a significant reduction in lumbar spine and femoral shaft bone density, and a non-significant reduction in femoral neck bone density. ${ }^{16}$ Again, the results were not confounded by measured lifestyle factors including diet, exercise, menopausal age, and body mass index.

Finally, an analysis of 544 men and 822 women in southern California ${ }^{17}$ reported sig- 
nificantly reduced BMD of the hip among men and women who smoked when compared with non-smokers. There was a dose-response relationship between baseline smoking status in this cohort study and hip BMD. However, smoking was not associated with reduced BMD at other skeletal sites. Our study supplements these observations and strongly suggests that the relationship between cigarette smoking and BMD cannot be entirely explained by the altered oestrogen status, diet, activity, and alcohol consumption, of cigarette smokers. The adverse effect of smoking was detected at both the lumbar spine and the hip, and was present among both men and women. Furthermore, duration of smoking had a significant effect on bone density at both the spine and hip among men and at the spine among women. The presence of a significant reduction in bone density at both sites among ex-smokers, when compared with current smokers, is in accord with the notion that cigarette smoking may adversely influence peak bone mass more than subsequent age-related bone loss. The precise physiological consequences of exposure to smoking remain to be elucidated. In the Australian twin study, ${ }^{16}$ smoking was found to be positively associated with higher serum concentrations of gonadotrophins, and lower concentrations of serum parathyroid hormone. These may be markers for a reduction in the circulating oestrogen level with a secondary increase in bone resorption. However, no direct association was found between measured oestrogen levels and tobacco use in the study.

The association we observed between cigarette smoking and the risk of oesteoporosis has important public health consequences. Among men, each decade of smoking is associated with a deficit in spinal bone density of $1.5 \%$ and hip bone density of $1.1 \%$. The corresponding values among women are $1.5 \%$ and $0.4 \%$. In the United States and Europe, around $25 \%$ of women smoke regularly and most middle aged and elderly female smokers began smoking during the first two decades of life. The results of this and previous studies suggest that cigarette smoking is associated with reduced bone density in later life, independently of potential confounding variables, and that programmes to reduce the burden of osteoporotic fractures in the general population should incorporate measures to reduce tobacco use among men and women.

This study was supported by project grants for the Wessex Medical School Trust and the Medical Research Council of Great Britain. We thank the subjects who participated in the study, and the nurses who performed the interviews and venepuncture. We also thank the Hertfordshire County Archives and the Hertfordshire health authorities who preserved the records and allowed us to use them, and the staff at the NHS Central Registry, Southport, and Office of Population Censuse and Surveys, London, who traced the men and women. Computing support was provided by Vanessa Cox and Paul Winter, and the manuscript was prepared by Katy Cuninghame.

1 Cooper C. Epidemiology and public health impact of osteoporosis. Bailliere's Clin Rheumatol 1993;7:459-77.

Cooper $\mathrm{C}$, Wickham C. Cigarette smoking and the risk of age-related fractures. In: Wald N, Baron J eds. Smoking age-related fractures. In: Wald N, Baron J eds. Smoking Press 1990;93-100.

3 Nguyen T, Sambrook P, Kelly P, Jones G, Lord S, Freund J Eisman J. Prediction of osteoporotic fractures by postura instability and bone density. BMF 1993;307:1111-14.

4 Law M. Smoking and osteoporosis. In: Wald N, Baron eds. Smoking and hormone-related disorders. Oxford, Oxford University Press 1990;83-92.

5 Hales CN, Barker DJP, Clark PMS, Cox LJ, Fall C, Osmond $C$, Winter PD. Fetal and infant growth and impaired glucose tolerance at age 64. $B M F$ 1991;303:1019-22.

6 Barker DJP, Meade TW, Fall CHD, Lee A, Osmond C Phipps K, Stirling Y. Relation and fetal and infant growth to plasma fibrinogen and factor VII concentrations in to plasma fibrinogen and factor
adult life. $B M \mp$ 1992;304:148-51.

7 Thomas M, Goddard E, Hickman M, Hunter P. General household survey 1992. OPCS Social Survey Division, London: HMSO, 1994

8 Cooper C, Barker DJP, Wickham C. Physical activity, muscle strength, and calcium intake in fracture of the proxima femur in Britain. BMF 1988;297:1443-6.

9 Wickham C, Walsh K, Cooper C, Barker DJP, Margetts BM, Morris J, Bruce S. Dietary calcium, physical activity and risk of hip fracture: A prospective study. $B M \mathcal{F} 1989$ 299:889-92.

10 Slemenda CW. Cigarettes and the skeleton. New Eng $\mathcal{F}$ Med 1994;330:430-1.

11 Johnell O, Nilsson BE. Life-style and bone mineral mass in perimenopausal women. Calcif Tissue Int 1984;35:354-6.

2 Sowers MR, Wallace RB, Lemke JH. Correlates of midradius bone density among postmenopausal women: radius bone density among postmenopausal women

13 Jensen GF. Osteoporosis of the slender smoker revisited by epidemiologic approach. Eur f Clin Invest 1986;16:239-42.

14 Krall EA, Dawson-Hughes B. Smoking and bone loss among postmenopausal women. $f$ Bone Min Res 1991;6:331-8.

15 Slemenda CW, Hui S, Longcope C, Johnston Jr CC. Cigarette smoking, obesity and bone mass. $\mathcal{F}$ Bone Min Res 1989;4:737-41.

16 Hopper JL, Seeman E. The bone density of female twin discordant for tobacco use. New Eng $\mathcal{F}$ Med 1994;330: 387-92.

17 Hodlenback KA, Barrett-Connor E, Edelstein SL, Holbrook T. Cigarette smoking and bone mineral density in olde men and women. Am ₹ Pub Health 1993;83:1265-70. 